\title{
Adiabatic sum-frequency generation in the visible region
}

\author{
Huseyin Cankaya, ${ }^{1,2^{*}}$ Anne-Laure Calendron, ${ }^{1,2}$ Haim Suchowski ${ }^{3}$ and Franz X. Kärtner, ${ }^{1,2,4}$ \\ ${ }^{I}$ Center for Free-Electron Laser Science, Deutsches Elektronen Synchrotron, Notkestrasse 85, 22607 Hamburg, Germany \\ ${ }^{2}$ Department of Physics and the Hamburg Center for Ultrafast Imaging, University of Hamburg Luruper Chaussee 149, D- \\ 22761 Hamburg, Germany \\ ${ }^{3}$ NSF Nano-scale Science and Engineering (NSEC), University of California, Berkeley, CA-94720, USA \\ ${ }^{4}$ Department of Electrical Engineering and Computer Science and Research Laboratory of Electronics, \\ Massachusetts Institute of Technology, Cambridge, Massachusetts 02139, USA \\ "Corresponding Author, e-mail: hcankaya@gmail.com
}

\begin{abstract}
We demonstrated adiabatic sum-frequency generation with $92 \%$ photon conversion efficiency between a white-light continuum and a strong pump at $1030 \mathrm{~nm}$ to cover the spectral range of 405-500 $\mathrm{nm}$ by using an aperiodically-poled nonlinear crystal.
\end{abstract}

(C)2013 Optical Society of America

OCIS codes: (320.7110) Ultrafast nonlinear optics; (320.7090) Ultrafast lasers; (190.4410) Nonlinear optics, parametric processes; (190.7170) Ultrafast nonlinear optics.

\section{Summary}

Nonlinear three-wave mixing provides an opportunity to generate light in wavelength regions that are not covered easily even with very broadband laser systems, such as Ti:Sapphire. The bandwidth of three-wave mixing inside a bulk crystal can be enhanced by decreasing the length of the medium at the expense of efficiency. Quasi-phase matching allows us to broaden the phase-matching bandwidth with relatively higher conversion efficiency [1]. However, also this process exhibits a trade off between bandwidth and efficiency. In the presence of a strong pump pulse, one can overcome this limit by employing Landau-Zener adiabatic transfer in non-linear optical wave-mixing. In this technique, the domain size of an aperiodically poled crystal varies slowly which allows the three-wave mixing process to follow an adiabatic passage process inside the nonlinear medium [2-4]. Previously, adiabatic sumfrequency generation (A-SFG) with photon conversion efficiencies as high as $11 \%$ for a seed-bandwidth of $80 \mathrm{~nm}$ centered around $765 \mathrm{~nm}$ was shown in Ref. [4]. In Ref. [3], a SFG conversion efficiency of $74 \%$ was recorded using a tunable seed source between 1400-1700nm. Recently, adiabatic difference frequency generation with almost $100 \%$ photon conversion efficiency was demonstrated inside a chirped A-PPLN crystal covering the mid-IR region $[5,6]$.

In this work, we describe and demonstrate adiabatic sum-generation (A-SFG) with $92 \%$ photon conversion efficiency inside an aperiodically-poled KTP crystal through Landau-Zener adiabatic. A slowly varying grating design inside the crystal allows us to convert white-light continuum in the range of $660-980 \mathrm{~nm}$ to $405-500 \mathrm{~nm}$ with very high photon conversion efficiency. To our knowledge, this is the highest photon conversion efficiency reported from a sum-frequency generation process.

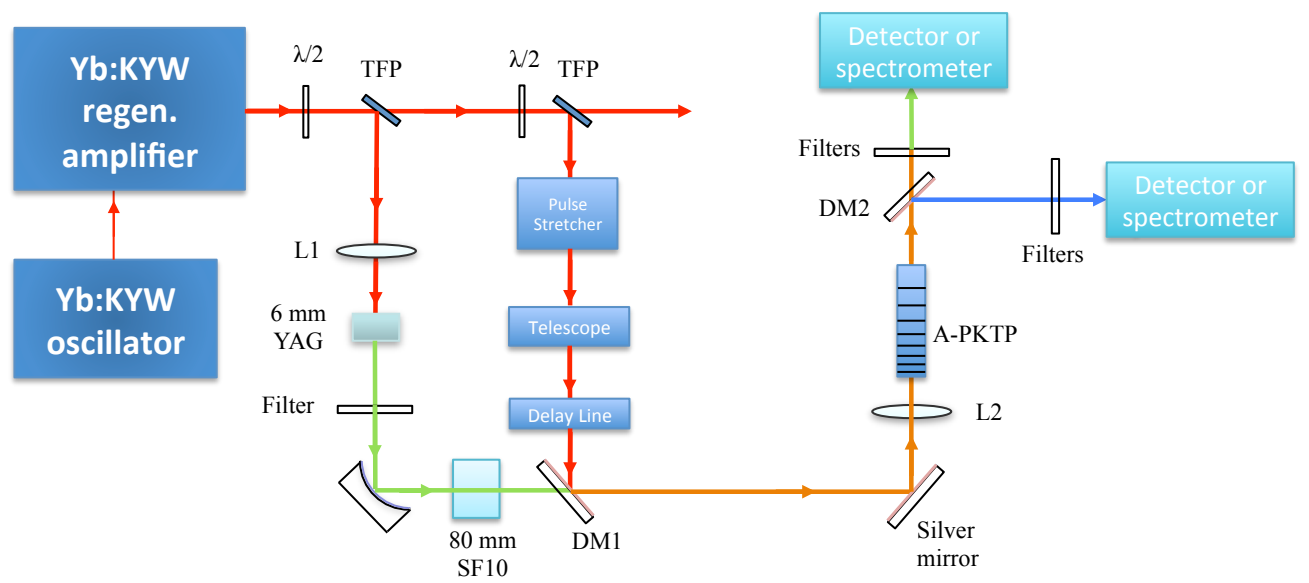

Fig. 1. Schematic of the adiabatic sum-frequency generation setup. The seed signal was generated via white-light continuum inside a YAG crystal. 
Figure 1 shows a schematic of our experimental setup. A Yb:KYW chirped pulse regenerative amplifier system (Amplitudexxxx) was used to generate both the signal and provide the pump beam in the A-SFG crystal. The regenerative amplifier was seeded by 250 -fs pulses generated by $\mathrm{Yb}: \mathrm{KYW}$ oscillator at $42.5 \mathrm{MHz}$ resulting in $1 \mathrm{~mJ}-$ pulses at $1 \mathrm{kHz}$ with pulse durations of about $450 \mathrm{fs}$. The output of the amplifier was split into two. The $1.5-\mathrm{mW}$ output was used to generate white-light continuum in a 6-mm YAG crystal. The beam was focused on the YAG crystal by using a lens (L1) with 100-mm focal length. Fine adjustment of the pump power was done with a variable attenuator. From the white-light process, we obtained spectra covering $510 \mathrm{~nm}$ and $2.5 \mu \mathrm{m}$. To test our aperiodicallypoled KTP crystal designed for the spectrum between 650 and $990 \mathrm{~nm}$ we extracted the corresponding continuum with a proper filter.

To avoid walk-off between seed and pump pulses, both pulses were stretched to about 10 ps. To stretch the pump pulses, a grating stretcher was employed which consists of two gratings with grove density of $1200 \mathrm{~mm}^{-1}$ separated by $12 \mathrm{~cm}$. On the other hand, the seed pulses were stretched by passing through $80-\mathrm{mm}$ long SF10 bulk glass. The signal and pump pulses were combined by using a dichronic mirror (DM1) and then focused inside the aperiodically poled KTP (A-PKTP) crystal by using a lens (L2) with focal length of $500 \mathrm{~mm}$. The poling period was varied between 2.1 and $5.6 \mu \mathrm{m}$ giving the phase matching for the signal wavelength range of $660-990 \mathrm{~nm}$. The chirp rate was determined by using the analysis for adiabatic frequency conversion in Ref's [2-3]. The length of the crystal was $20 \mathrm{~mm}$. The signal pulse energy was measured to be $6.5 \mu \mathrm{W}$ before the crystal. To determine the efficiency of the conversion, the depletion of the signal was measured both with a photo-detector and a spectrometer and compared with the measurement in the absence of the pump light. During the efficiency measurement, a couple of band-pass filters are used to avoid the pump and parasitic second-harmonic signal (SHG) at $515 \mathrm{~nm}$ on the detector or spectrometer. The SFG signal was separated by using a dichroic mirror (DM2). In the SHG measurement arm, the parasitic SHG signal was suppressed by using a low pass filter.
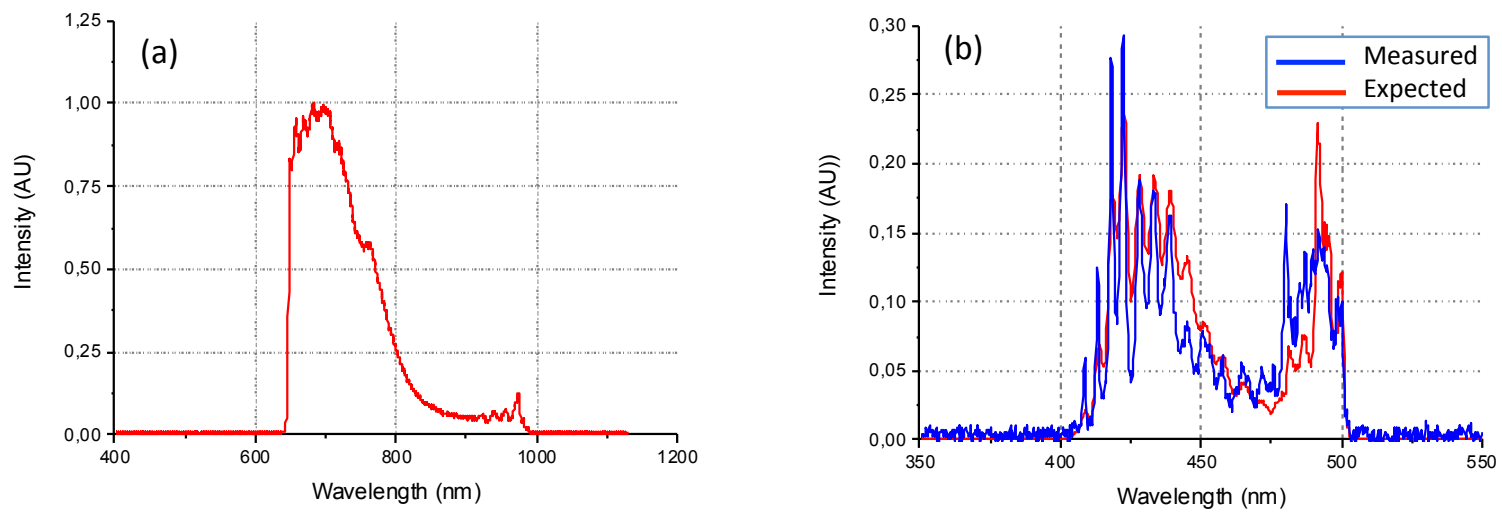

Fig. 2. (a) Spectrum of white-light continuum before the crystal. The spectrum was filtered to match the design of the crystal. (b) The spectra measured and expected of SFG signal.

Figure 2 (a) shows the spectra of the white light continuum before the crystal, which is filtered to match the design signal wavelength range of the chirped KTP crystal. The spectrum of the white-light covers the range between $660 \mathrm{~nm}$ and $990 \mathrm{~nm}$. In Figure 2 (b), the measured and expected spectra of the SFG signal after short pass filters are shown. The filters are used to filter out the parasitic SHG signal resulting due to strong pump at $1030 \mathrm{~nm}$. The expected spectrum was estimated by taking into account of the transmission the short pass filters. The ripples in the SFG spectrum were due to transmission spectrum of the filters used during the measurement. The spectra were measured by using HR 4000 Ocean Optics spectrometer. 

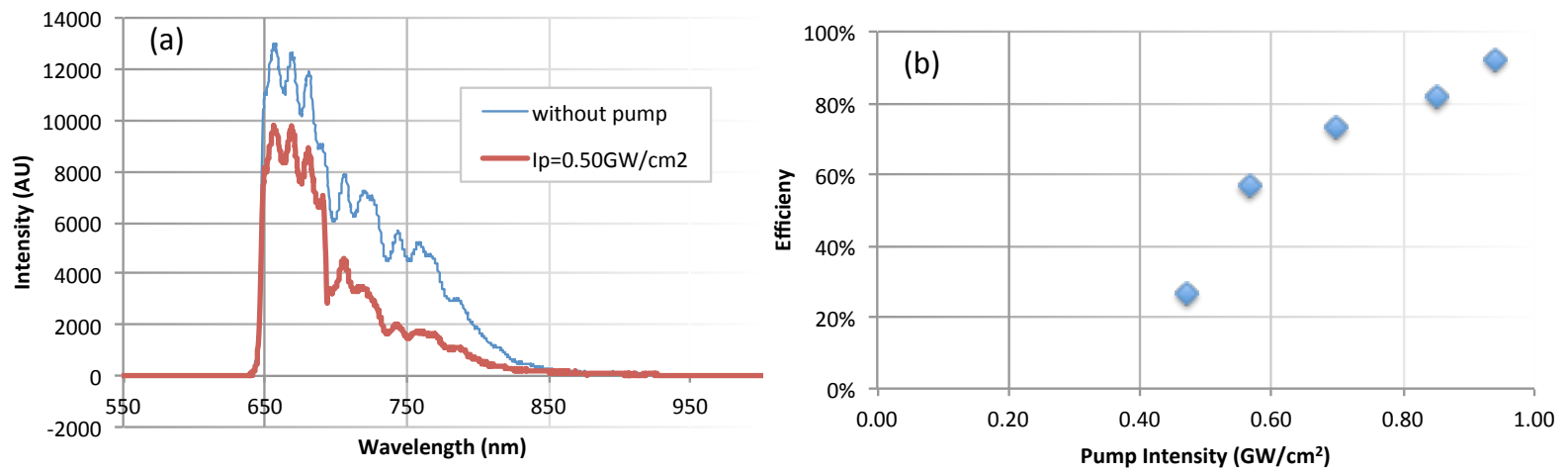

Fig. 3.(a) Spectra of signal in the absence and presence of pump light with intensity of $0.50 \mathrm{GW} /$ (b) $\mathrm{cm}^{2}$ Photon conversion efficiency in A-SFG process as a function of pump power..

Figure 3 (a) shows the spectrum of the white-light continuum after filters under two different regimes where there is no pump and the pump is intensity is about $0.50 \mathrm{GW} / \mathrm{cm}^{2}$. As can be seen from the figure, with the presence of the pump pulse, almost uniform spectral depletion was observed. The modulations in the spectra were due to filters used to cut the pump and residual second harmonic resulted due to strong pumping. Figure 3 (b) shows the efficiency as a function of pump intensity. When the pump intensity increased up to $0.94 \mathrm{GW} / \mathrm{cm}^{2}$, the SFG photon conversion efficiency increased up to $92 \%$. Above that, we observed damage on the surface of the crystal.

In conclusion, we experimentally demonstrated highly efficient sum frequency generation via Landau-Zener adiabatic transfer in an aperiodically poled KTP crystal for the signal range of 660-990nm. The photon conversion efficiency was measured to be $92 \%$ for a very broad spectral range 405-500 nm when the pump intensity was close to $1 \mathrm{GW} / \mathrm{cm}^{2}$.

\section{References}

[1] M. Arbore, A. Galvanauskas, D. Harter, M. Chou, M.M. Fejer, "Engineerable compression of ultrashort pulses by use of second-harmonic generation in chirped-period-poled lithium niobate," Opt. Lett. 22, 1341 (1997).

[2] H. Suchowski, D. Oron, A. Arie, and Y. Silberberg, "Geometrical representation of sum frequency generation and adiabatic frequency conversion," Phys. Rev. A, 063821 (2008).

[3] H. Suchowski, V. Prabhudesai, D. Oron, A. Arie, and Y. Silberberg, "Robust adiabatic sum frequency conversion," Opt. Express 17, 12731 (2009).

[4] H. Suchowski, B. D. Bruner, A. Ganany-Padowicz, I. Juwiler, A. Arie, and Y. Silberberg, "Adiabatic frequency conversion of ultrafast pulses," Appl. Phys. B 105, 697-702 (2011).

[5] J. Mosses, H. Suchowski and F.X.Kaerner, "Fully efficient adiabatic frequency conversion of broadband Ti:sapphire oscillator pulses," Opt. Lett. 37, 1589 (2011).

[6] H. Suchowski, P. R. Krogen, S.W. Huang, F.X. Kaertner, and J. Moses, "Octave-spanning Coherent Mid-IR Generation via a Single Adiabatically Chirped Grating78," CLEO, San Jose (2013) 